\title{
FORUM
}

\section{Trend Analysis of Research on Informed Consent in Clinical Trials : Comprehensive Retrieval via Electronic Databases}

\author{
Taichi HATTA ${ }^{* 1}$, Toshinori MURAYAMA*1,*2, Keiichi NARITA*2, \\ Eriko SUMI*1,*2 and Masayuki YOKODE ${ }^{* 1, * 2}$ \\ *1 Department of Clinical Innovative Medicine, Kyoto University Graduate School of Medicine, Kyoto, Japan
}

*2 Translational Research Center, Kyoto University Hospital, Kyoto, Japan

\begin{abstract}
Informed consent (IC) is an essential ethical requirement for conducting clinical trials. This study aimed to investigate changes in research on IC for clinical trials and compare the trend in Japan with that overseas.

In February 2010, the electronic databases, PubMed and Japana Centra Revuo Medicina were searched for studies on IC for clinical trials both in and outside Japan.

The literature search identified 89 Japanese studies and 401 overseas studies concerning IC for clinical trials. In Japan, these articles were first published in 1993, and the number increased remarkably from 2002. Many of these articles discussed the contribution of a clinical research coordinator or the understanding of the information by trial subjects. The overseas studies were first published in the early 1980s, and the number increased remarkably in the 1990 s.

Since, the enforcement of the new Good Clinical Practice in 1998, observational studies on IC for clinical trials have been conducted in Japan. However, most of these studies applied a quantitative approach, and qualitative analysis is limited. In Japan, IC is not focused exclusively on the protection of trial subjects, but also serves as the basis for an important encounter to build the physician-patient relationship. Therefore, investigations of the IC process by qualitative and quantitative approaches are necessary.
\end{abstract}

Key words : informed consent, clinical trial, research ethics

\section{Introduction}

Informed consent (IC) is an essential ethical requirement for conducting clinical trials. Originating in the US, the concept of IC was introduced in Japan in the $1980 \mathrm{~s}^{1}$. Initially, this concept failed to take full root because of its novelty, given the different medical and cultural backgrounds. In the 1990s, research suggested that the physician-patient relationship should be discussed in order to study the nature of the IC process in Japan ${ }^{2)}$. However, before the social/cultural environment was fully matured, IC was legally required for the conduct of clinical trials according to the adoption of ICH-GCP in $1998^{3)}$. In the modern era of increasing globalization, the historical and cultural backgrounds have to be reviewed so as to understand the problems surrounding IC in Japan. This will also inevitably lead to discussions about the methodology of observational study for IC.

The objectives of this study were to investigate the trends in investigations on IC for clinical trials, to review the studies on IC for clinical trials in Japan, and to compare the IC studies in Japanese clinical trials with those overseas.

\section{Methods}

1) Search of literature: Comprehensive retrieval via electronic databases

To examine the number of published papers on IC for clinical trials, we searched PubMed and Japana Centra Revuo Medicina (http://login.jamas.or.jp/enter.html), the latter being an electronic search engine for medical journals written in Japanese. Searches were originally conducted in December 2007, and repeated in February 2010.

To search for Japanese papers on IC for clinical trials, Japana Centra Revuo Medicina was searched using the retrieval terms "informed consent", "clinical trials" and "data collection", and PubMed was searched using "informed consent", "clinical trials", "data collection" and "Japan". Meanwhile, to search for overseas publications, PubMed was searched using the retrieval terms "in-

Address for correspondence : Murayama T. Translational Research Center, Kyoto University Hospital, 54 Shogoin-Kawaharacho, Sakyo-ku, Kyoto 606-8507, Japan

(Manuscript received March 31, 2010 ; revised July 16, 2010 and October 4, 2010 ; accepted October 24, 2010) 


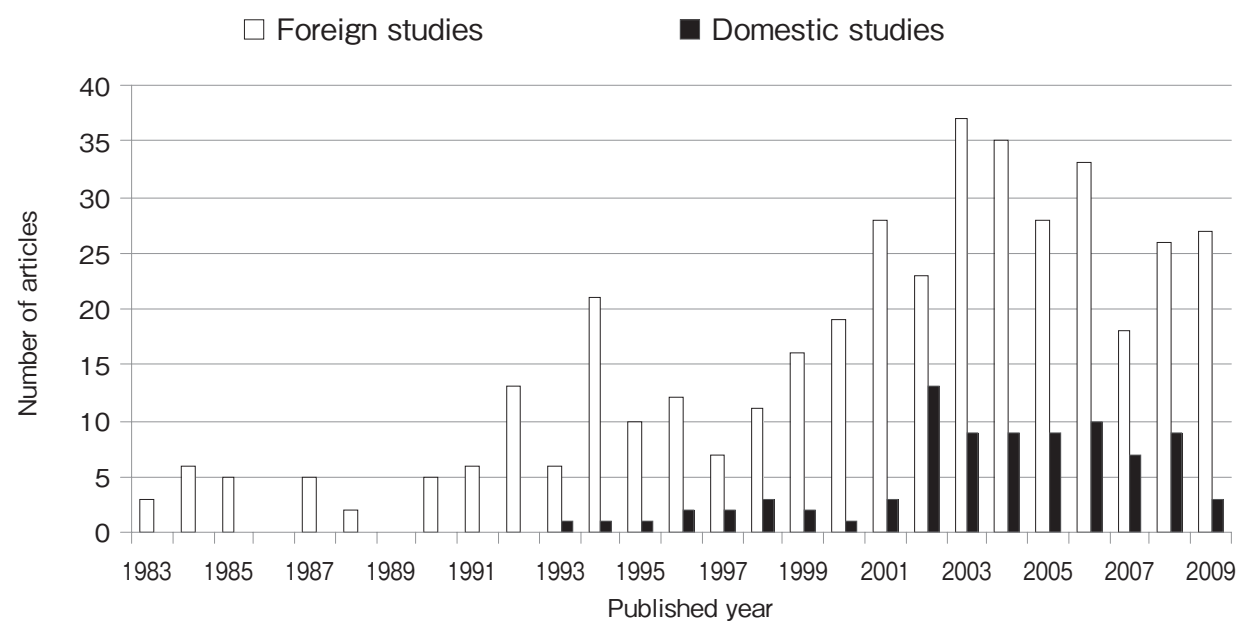

Fig. The number of IC studies for clinical trials

formed consent", "clinical trials" and "data collection”, and those identified in the search of Japanese papers were excluded.

2 ) Extraction and investigation of observational studies on IC in Japan

Japanese studies with abstracts were checked to see whether they were observational studies on IC for participation in clinical trials, which used a questionnaire or an interview with patients, doctors or co-medicals. IC studies without abstracts were excluded because the contents could not be screened, and also most of them were conference proceedings or reviews. Moreover, studies related to decision making in general medicine or studies containing only discussion on the significance of IC for clinical research were also excluded. Then, the selected papers were classified according to the theme of the article and the method used in the study.

\section{Results}

1) Number of articles identified under each of the search conditions by year

Comprehensive retrieval via electronic databases identified 89 Japanese studies. There was no duplication of data between Japana Centra Revuo Medicina and PubMed. Four hundred and one overseas studies were identified. Figure shows the studies categorized by year of publication.

Japanese research was first reported in the 1990s, and the number increased greatly from 2002. On the other hand, overseas studies were first published in the early 1980s, and the number increased remarkably in the 1990s (Fig.).

\section{2 ) Extraction and investigation of observational studies on IC in Japan}

Thirty-two of 89 Japanese studies had abstracts. Among the 32 articles, 17 that reported observational studies on IC for participation in clinical trials using a questionnaire or an interview of patients, doctors or co-medicals were extracted $^{4-20)}$. Most of them dealt with IC for trials for new drug application (NDA).

Six of the 17 articles reported on 'the contribution of a clinical trials coordinator (CRC) in clinical trials ${ }^{\text {, }}{ }^{4,611-13,16)}$, 6 reported on IC assessment, such as the level of understanding of clinical trials ${ }^{\prime, 9,10,14,15,20)}$, and the others studied aspects of IC for clinical trials, such as preference of physicians to recruit participants ${ }^{5,17}$, perception of pediatric nurses with regard to competency $^{8}$, use of videotape instruction for clinical trials ${ }^{18)}$ and communication training for $\mathrm{CRC}^{19)}$.

Articles on 'the contribution of a CRC in clinical trials' revealed the factors that participants and physicians recognized as merits in the clinical trials and their impressions of a $\mathrm{CRC}^{4,6,11-13,16)}$. These articles reported that a CRC contributed mainly to communication with participants, such as drug administration guidance ${ }^{4)}$, care for participants ${ }^{4,11)}$ and help in decision-making processes ${ }^{12,13)}$. Other articles described the role of a $\mathrm{CRC}$ or the expectations of physicians on a $\mathrm{CRC}^{6,16)}$.

Articles on 'the level of understanding of clinical trials' reported how well trial participants understood the clinical trials after receiving an explanation ${ }^{7,9,10,14,15,20)}$. The responses suggested that most of the participants could understand the trials if they were given a full explanation. The aspects cited by the participants as difficult to 
understand were 'the significance of a placebo' ${ }^{10)}$, 'doubleblinded study' ${ }^{10,14)}$, 'randomized controlled study' ${ }^{14)}$ and 'main effect and side effects' ${ }^{7,9,10,14,15,20)}$.

When the 17 articles were categorized according to study method, the vast majority adopted a quantitative approach, while only one employed a qualitative approach $^{12)}$. Nakamura et al. took the verbatim record of IC communication between patients and CRCs, and conducted content analysis, which is a standard methodology in social sciences for studying the content of communication $^{12)}$.

\section{Discussion}

\section{1) Relation between domestic IC articles and Japa-} nese social background

We included both articles and conference proceedings (with abstracts) on IC in the present analysis. Although the significance of each type of reference remains to be discussed, we considered them to be equal in this trend analysis. The rationale is that our purpose was to investigate the trends of research on IC for clinical trials, and if conference proceedings were excluded, the retrieved data might be too small to be analyzed properly and the trend might be biased.

In this study, a search of literature using electronic databases found that research on IC for clinical trials had been published in Japan since 1993, and the number increased remarkably from 2002. Many articles concerning IC for clinical trials discussed the contribution of a CRC or the understanding of information provided to trial subjects. These results might be related to the Japanese social background factors such as regulation and infrastructure regarding clinical trials. Specifically, it was a major task for institutions to train CRCs initially, and CRC developed rapidly as a new occupation to support clinical trials including IC.

Although IC started to be practiced substantially in Japan in the early $1980 \mathrm{~s}^{1)}$, this study indicates that the focus on IC for clinical trials started later. One of the possible reasons for this delay is that the procedure of IC for general medical practice was different from that for clinical trials. It seems that IC was not regarded as a subject of observational study, but the discussion on IC mainly concerned the disclosure of diagnosis to cancer patient in the $1980 \mathrm{~s}^{2)}$.

In the 1990s, some Japanese IC studies were retrieved by our research. With the adoption of ICH-GCP, the Pharmaceutical Affairs Law and the new GCP ordinance for NDA trials were fully enforced in 1988 and training courses for CRC organized by the Ministry of Welfare were started $^{3)}$. Since then, medical institutions that conduct NDA trials have set up clinical trial management centers and institutional review boards (IRBs) ${ }^{*}$, and organize systems that include personnel. There was probably an increased awareness of the importance of protecting the rights and well-being of trial subjects since the enforcement of ICH-GCP and the cooperation of CRC in clinical trials. However, it was probably not until written consent became a legal obligation for pharmaceutical developers and medical institutions that research on IC for clinical trials was started in full swing.

The Japanese articles began to increase in number greatly from 2002. In the 2000s, the Declaration of Helsinki was amended several times ${ }^{21}$, and some Japanese guidelines on medical research were also revised by ministries, agencies and medical societies ${ }^{22,23}$. As mentioned in the Declaration of Helsinki, each potential subject should be adequately informed of the aims, methods, sources of funding, any possible conflicts of interest, institutional affiliations of the researcher, the anticipated benefits and potential risks of the study and the discomfort it may entail, as well as any other relevant aspects of the study ${ }^{21)}$. During these changes surrounding clinical trials, several medical institutions built up their own infrastructures for clinical trials, and they accumulated and reported their experiences. Hence, the Japanese articles mainly focused on the contribution of $\mathrm{CRC}$ and the understanding of participants.

\section{2 ) How should the quality of IC be evaluated?}

From the viewpoint of IC quality, studies worldwide have mainly focused on participant understanding ${ }^{25-27}$. Our results also showed the same tendency in $\operatorname{Japan}^{7,9,10,14,15,20)}$. This might have been influenced by the Declaration of Helsinki ${ }^{21}$. However, the extent of participant understanding is not always related to the process of IC or IC itself. The IC process is an individual issue and a subjective decision-making process, and contains psychosocial aspects such as medical culture and physicianpatient relationship. Therefore it is difficult to identify all the factors associated with IC. In order to evaluate the process of IC in clinical trials, qualitative approaches would be useful, which include observation, interviews or verbal

${ }^{*}$ IRBs : To protect the rights of participants, IRBs have been set up in several hospitals in Japan since early in $1980 \mathrm{~s}^{24)}$. After adoption of ICH-GCP, the Pharmaceutical Affairs Law and the new GCP ordinance ${ }^{3)}$ empowered the function of IRBs. 
interactions and focus on the meanings and interpretations of the participants ${ }^{28)}$. To illustrate this approach, a representative article found by our comprehensive search is described below.

To reveal how CRC approached participants during IC, Nakamura et al. ${ }^{12)}$ took the verbatim records of IC communication between patients and CRCs. They conducted content analysis with the verbatim records, and categorized the approaches into 8 patterns including 'setting a good IC environment for participants and CRC,' 'providing information about the clinical trial' and 'estimating the stance of participants'. This is a pioneering IC study with a qualitative method in Japan.

The qualitative approach, which is not yet popular in IC evaluation, could reveal how gaps are formed between participants and medical professionals during the IC process. Therefore, to evaluate IC for clinical trials, we have to start to observe the process of IC in a multifaceted manner, namely, quantitatively and qualitatively.

\section{3 ) Limitations of this study}

Although we conducted a thorough search by comprehensive retrieval via electronic databases in order to identify articles on IC in clinical trials, our method has several limitations : 1) A database does not necessarily provide all the target articles that we would expect given the search conditions. Indeed, several articles dealing with IC for clinical trials in Japan were missed in our comprehensive retrieval $^{29-32}$. 2) We excluded reports that did not contain abstracts. Therefore significant conference proceedings could have been overlooked.

\section{Conclusion}

After the enforcement of the new GCP, observational studies on IC for clinical trials have been conducted in Japan. However, most of them applied a quantitative research approach, and qualitative analysis of IC for clinical trials is limited. In Japan, IC is not aimed exclusively for the protection of trial subjects, and it is necessary to investigate the IC process by both qualitative and quantitative approaches.

\section{Acknowledgments}

This study was partly supported by Health Labor Sciences Research Grant and Grant-in-Aid for Scientific Research (C) from Japan Society for the Promotion of Science.

\section{References}

1) Shimoyama M. Informed Consent-historical evolution and recent trend-. The Journal of Clinical Science. 1997 ; 33(4) :38997 (in Japanese).

2) Yanagida K. Inspiring Informed Consent. Tokyo : Chuohoki Publishers, 1996 (in Japanese).

3) Good Clinical Practice (Amended March 27, 1997). Ministry of Health and Welfare.

4) Okazawa K, Takano M. Test subjects' perception of clinical trials of drugs and pharmacist clinical research coordinators' responsibilities : a questionnaire survey. JJpn Assoc Rural Med. 2007 ; 56 (1) : 22-8 (in Japanese).

5) Fukui T, Rahman M, Morita S, Sakamoto J. Informed consent in the Candesartan Antihypertensive Survival Evaluation in Japan (CASE-J) trial : a survey of collaborating physicians. Hypertens Res. $2006 ; 29(7): 471-4$.

6) Tsuji T, Wakasugi Y, Kanaya A, Yamasaki M, Nishida T, Kikutake K, et al. Questionnaire survey of physicians to assess their appreciation of clinical research and evaluate CRC practice in Kyushu University Hospital. Jpn J Pharm Health Care Sci. 2006 ; 32 (2) 164-73 (in Japanese).

7) Asada R, Noda K, Sakiyama E, Morita E, Sato T, Nishio M, et al. Survey of participants to clinical trial in Fukuoka University Hospital : Relationship between the participant's understanding of informed consent and their feeling of unease for clinical trials. Medical Bulletin of Fukuoka University. 2006 ; 33 (1) : 25-9 (in Japanese).

8) Matsuda I, Ooiwa Y, Fujimura M, Yoshida I, Ito S, Tsujimoto G, et al. A questionnaire concerning pediatric health care administered to pediatric nursing managers. Jpn J Dev Pharmacol Ther. 2003 ; 16 (1) : 123-34 (in Japanese).

9) Ando Sachiko, Ando Shoko, Kato T. Level of subject understanding of information in a clinical trial. J Jpn Soc Nurs Res. $2003 ; 26$ (4) : 99-108 (in Japanese).

10) Watanabe K, Hara N, Negishi H, Sonehara W, Watanabe $Y$. Current situation and issues of taking consent of IND trials in National Defense Medical College, according to questionnaire to participants and physicians. JournaI of the National Defense Medical College. $2002 ; 27$ (4) : 184-90 (in Japanese).

11) Terada A, Kajiyama M, Nakatani S, Kashiwaguma R, Tezuka K, Kobayashi S, et al. Questionnaire for investigator and subject on clinical research coordinator in St. Marianna University Hospital. Jpn J Clin Pharmacol Ther. 2002 ; 33(5) : 219-26 (in Japanese).

12) Nakamura N, Sakamoto T, Obara I, Nakao M, Morishita N, Naito $\mathrm{M}$, et al. The methods for explanation of NDA trials by CRCs. Quality Nursing. 2002 ; 8(7) : 619-24 (in Japanese).

13) Tanaka R, Ogawa S, Iizuka $Y$, Oomura M, Hayase N, Matsubara $\mathrm{K}$, et al. The utility of research nurse during the process of informed consent for IND trials. Jpn J Nursing Arts. 2002 ; 48 (7) : 856-60 (in Japanese).

14) Nakano S. Reality of informed consent for IND trials in Japan, results of questionnaire comparing the participants of doubleblinded comparative study in Japan with physicians-in-charge of them.Jpn Pharmacol Ther. 1997 ; 25 (9) : 2223-47 (in Japanese).

15) Kitajima K, Nakamura $Y$, Yao S, Yanagida T, Nakamura J, Tsubakihara Y, et al. Inquiry of patients about informed consent in clinical experimentation - the case of recombinant human erythropoietin on renal anemia in predialysis patients-. Medical Journal of Osaka Prefectural Hospital 1995 ; 18 (1) : 63-6 (in Japanese). 
16) Yanagawa $H$, Nishiya $M$, Miyamoto $T$, Shikishima M, Imura M, Irahara M, et al. Clinical trials for drug approval : a pilot study of the view of doctors at Tokushima University Hospital. $J$ Med Invest. $2006 ; \mathbf{5 3}(3-4): 292-6$.

17) Rahman M, Morita S, Fukui T, Sakamoto J. Physicians' reasons for not entering their patients in a randomized controlled trial in Japan. Tohoku J Exp Med. 2004 ; 203(2) : 105-9.

18) Ishii $M$, Ohashi $Y$. Influence of an educational videotape on attitudes toward participating in cohort studies : results of a randomized controlled trial. Nippon Koshu Eisei Zasshi. 2007 ; 54 (7) : 419-26.

19) Arita E, Iioka T, Ujihara A, Omori R, Koshiba S, Kamata S, et al. Evaluation of program to develop communication skills for health care professionals-Role-playing exercises introduced for training in informed consent process in clinical trials-. Jpn J Pharm Health Care Sci. $2008 ; 34(8): 727-35$ (in Japanese).

20) Nishiwaki H, Yasuda I, Hayashi H. A study on informed consent in clinical trial. J Hokkaido Rural Med. 2009; 41:29-33 (in Japanese)

21) The Declaration of Helsinki (2008). World Medical Association.

22) The Ethical Guidelines for Clinical Studies (Amended July 31, 2008). Ministry of Health, Labour and Welfare, Japan.

23) The Ethical Guidelines for Epidemiology Studies (Amended August 16, 2007). Ministry of Education, Culture, Sports, Science and Technology, and Ministry of Health, Labour and Welfare, Japan.

24) Good Clinical Practice (October 2, 1989). Ministry of Health and Welfare.
25) Sugarman J, McCrory DC, Hubal RC. Getting meaningful informed consent from older adults: a structured literature review of empirical research. J Am Geriatr Soc. 1998; 46 (4) :51724.

26) Joffe S, Cook EF, Cleary PD, Clark JW, Weeks JC. Quality of informed consent in cancer clinical trials : a cross-sectional survey. Lancet. $2001 ; 358$ (9295) : 1772-7.

27) Flory J, Emanuel E. Interventions to improve research participants' understanding in informed consent for research : a systematic review. JAMA. 2004 ; 292 (13) : 1593-601.

28) Holloway I, Wheeler S. Ethical issues in qualitative nursing research. Nurs Ethics. 1995 ; 2(3) : 223-32.

29) Inoue Y, Kamiya E, Tachibana I, Yamazaki E, Atagi S, Kawahara M. Role of nurses in offering informed consent to clinical trials for advanced lung cancer.Jpn J Lung Canc. 2000 ; 40 (7) : 719-23 (in Japanese).

30) Takishita S. Large scale clinical trials for hypertention in Japan : obstacles in performing the trials. Ryukyu Med J. $2001 ; 20$ (4): 167-71 (in Japanese).

31) Kamei H, Ando S, Kato T, Naruse $Y$, Ichino M, Nabeshima T, et al. The role of clinical research coordinators on the quality control in clinical trials. Jpn J Clin Pharmacol Ther. 2003 ; 34(1): 19-23 (in Japanese).

32) Itoh K, Sasaki Y, Fujii H, Ohtsu T, Wakita H, Igarashi T, et al. Patients in phase I trials of anti-cancer agents in Japan; motivation, comprehension and expectations. Br J Cancer. 1997 ; $76(1)$ : 107-13. 